\title{
Engineering Lessons Learned in the Assembly, Commissioning, Initial Operation and in the Further Upgrading of Wendelstein 7-X
}

\author{
Lutz Wegener and the W7-X team, \\ Max Planck Institute for Plasma Physics, EURATOM Association
}

\begin{abstract}
Wendelstein 7-X (W7-X) was constructed from 2005 until 2015 at the Max-Planck-Institute for Plasma Physics in Greifswald, Germany. W7-X is one of the world largest stellarator, is five-fold symmetric, has a superconducting magnet system, and weighs about 1000 tons. W7-X started its first operation in 2015 with up to 4.3 MW ECRH and a heating duration of up to $6 \mathrm{~s}$, and injected energy per pulse up to $4 \mathrm{MJ}$. The injected energy per pulse was increased up to $200 \mathrm{MJ}$, for example $100 \mathrm{~s}$ at $2 \mathrm{MW}$ and also $30 \mathrm{~s}$ at $5 \mathrm{MW}$ in the third operation campaign in 2019. The machine was upgraded twice since 2016 and a third upgrading phase is carried out now. The main aim of upgrade is the increase of the plasma energy as well as the increase of the heating energy flow through the machine. That requires among others the installation of a High Heat Flux (HHF) Divertor and the strengthening of the associated cooling system. This contribution provides a general overview of the construction of W7 X. It summarizes some engineering lessons learned in the construction phases and in the first operation campaigns $($ OP1.1, 1.2a and 1.2b). Furthermore, the paper explains technology challenges as well as modifications due to the first experiences in commissioning and operation. Technical, organizational, and schedule aspects of the assembly are discussed as well.
\end{abstract}

Index Terms - Assembly, lessons learned, Wendelstein 7-X (W7-X).

\section{INTRODUCTION}

$\mathrm{W}$ $7-\mathrm{X}$ is a superconducting machine with 50 non-planar and 20 planar coils. A coil weighs about 5 tons and has an average diameter of $3.5 \mathrm{~m}$. The coil shapes were determined by an iterative computational process to closely reproduce the desired optimized magnetic field shape. The plasma vessel and its add-on components follow closely the toroidal, twisted shape of the magnetic flux surfaces. The magnet system is subdivided into five identical modules, which allows to some extent a parallel assembly sequence. The helically shaped Plasma Vessel (PV) and the Outer Vessel (OV) form the cryostat (16 $\mathrm{m}$ outer diameter), which envelops the $4 \mathrm{~K}$ cryogenic magnet system. Cryostat vessel walls are covered with multi-layer insulation and cryo-shields. 250 ports connect the openings between both vessels. All in-vessel components follow the helical shape of the PV. The high packing density of the components and their helically bent shape cause a high effort in the assembly and a comprehensive as-built customization. First assembly planning started around the year 2000 and the last upgrade of the in-vessel installation is planned to be accomplished in 2021. W7-X is not a nuclear facility; however, due to the operation with deuterium and due to the occurrence of minor radiation, German radiation safety regulations have to be respected in the design, construction and operation.

\section{WORK-FLOW AND STRUCTURE IN THE ASSEMBLY DIVISION (AS)}

The main assembly phase between 2005 and 2015 required about 920,000 direct person-hours and about 550,000 personhours for the associated engineering assistance (work preparation, technology development, metrology, leak testing, welding specialists, consultancies). The assembly division is subdivided into three departments: device assembly (AS-DA), work preparation and planning (AS-AV), and technology (AST). AS-DA comprised all work force doing the assembly work on-site in a shift system and with the associated middle management. That includes on-site work-packages for metrology, leak-testing and welding. Prior to their use, all installation work-packages are sub-structured in individual steps (so-called Quality Assurance and Assembly Plans QAAP) through AS-AV and hold points and information points for the Quality Assurance (QA) are added. Among others, the technical responsible officer of the component (RO) and the quality management (QM) check and release these QAAPs prior to the use. Every craftsman signs his work-step in the QAAP once fulfilled. That includes notes on quality deviations, non-conformity reports (NCRs), stop cards etc. This system ensures a full traceability of all assembly work. In case of quality deviations, RO and QM have to countersign proposed correction and repair measures prior to their execution. Basis for the QAAP are work instructions (WI), which are produced by AS-T in advance. WI must be compatible with the requirements of the design and the integration, which are determined by the engineering department (EN) and the RO. The RO, QM and other directly involved project-teams check and release the WI prior to its use. They make sure that the EN requirements have been properly considered. Work Instructions 
contain descriptions of tools and equipment to be used, partlists, drawings, references to CAD-models and other specific boundary conditions, which must be considered during the assembly of components. Tasks of AS-T are the qualification of assembly processes, the procurement of tooling and equipment and also the definition of guarantee-values. AS-T prepares and procures dummies for assembly-simulations and it develops the test-instructions and organizes the associated test-sequences and trials. E.g., all accuracy relevant assembly steps with the potential of an increased risk were mocked-up. This applies particularly to almost all welding processes. Personnel from AS-DA carries out the tests under the supervision of AS-T. Tests are used not only to develop feasible assembly procedures and to identify achievable guarantee values, but also to train the personnel and to define required process times. Test sequences are embedded in the daily assembly work program through AS-AV, depending on the available resources and the given priorities. In terms of the onsite work-progress, AS-AV is responsible not only for the timely availability of assembly documents but also for the monitoring of the achieved progress and keeps the assemblyplan up-to-date. AS-AV checks the progress in every shift together with the line-management. This monitoring enables early mitigation-measures if assembly work packages begin to delay. Within AS-DA, an assembly-service team makes sure that all materials, components and small parts are available in the right place at the right time. Up to 15 engineers have been working in AS-T and up to 8 in AS-AV. In peak periods about 150 workers, technicians and engineers worked in AS-DA, i.e. altogether up to 170 staff were occupied in the Assembly Division. Two thirds of all needed work force in the assembly were hired from industrial firms. They were fully integrated in the assembly hierarchy and mixed teams were formed. One third of the needed work force for the assembly was personnel of the institute. These personnel was made available through a temporary construction-oriented restructuring of the existing organization.

From the beginning of the assembly, work-safety played a decisive role. Narrow working conditions and many parallel work packages at the same place represent a source of increasing dangers at any time. A central safety manual summarizes all safety instruction, hazard analyses and protection measures. This manual was one of the first formal documents in the assembly. An on-site coordinator (OSC) cares for safe working conditions and keeps the safety manual up-todate. The OSC carries out all initial general safety instructions. In addition, the personnel who works in the assembly area gets work place related safety instructions three to four times per year. A live and practical instruction style ensures the attention of the participants and the sustainability of the contents.

A special challenge is the simultaneous work of the device assembly and the device commissioning. Parts of the facility are already under pressure or under electrical power. Dangerous areas are separated with colored ribbons. Each color represents a specific class of danger. These closed areas may not be entered. Work activities which could extend into these areas, require special permission. The level of danger on-site can change at any time. Therefore, the shift supervisors keep the safety information at the entrance of the hall up-to-date. The department heads must permit, in writing, any work at components, which are under commissioning. The shift supervisor is part of this permission chain. These precaution measures did hamper the daily progress somewhat, but it was the best compromise between assembly progress and safety. There was no severe accident up to now.

\section{SCHEDULE ASPECTS}

The head of the Assembly Division elaborated the first resource loaded assembly schedule in 2003, which showed an assembly end in 2010. At that time the component design, the assembly tooling and the assembly processes were mature for the major steps of the mechanical pre-assembly of magnet modules. This work package comprised about $20 \%$ of the entire scope of the main assembly. However, many smaller but also decisive steps and procedures for the mechanical pre-assembly were not yet qualified. Eighty percent of the schedule basis were best-guess estimates and hence, the schedule was rather uncertain. The maturity of both, component designs and associated assembly processes, evolved gradually during the entire assembly phase. Limitation in experienced, trained, and suitable human resources, but also the necessity to develop iteratively prototypes for processes and components, made this enduring evolution process unavoidable. In addition, the detailed design of W7-X and a systematic design review process grew only gradually together with the entire project. The assembly process started in March 2004 with the first delivered coil. From March 2005 onwards, the bottleneck in the delivery of the coils was fully overcome and the two-shift system was established in the Assembly Division. The forecast of the entire assembly work and the schedule itself became stable only after 2006. At this time, the estimated assembly end was in March 2014. Part of this stabilization was the introduction of an assembly-contingency of about one year. The actual end of the main assembly (last cables and pipes) was achieved in March 2015. That did not influence the start of the first plasma severely since the commissioning of W7-X started earlier and overlapped with the final assembly steps, which was not foreseen in the initial planning of 2006. After 2015, the construction of $\mathrm{W} 7-\mathrm{X}$ is completed in three upgrade phases as described in chapter five.

The AS-planning is updated comprehensively every six months in preparation of the regularly meeting of the project council. That applies also to the needed assembly budget. On a weekly basis, AS-AV optimized the assembly schedule and reported the construction progress to the senior project management.

\section{Challenges IN THE ASSEMBLy TECHNOLOGY}

One of the biggest costs and time driver in the assembly was (and is) the tightly packed design of W7-X in combination with the accuracy requirement (better than $10^{-4}$ ). The situation would have been more relaxed if clearances between components in the detailed design could be made at least $50 \mathrm{~mm}$ instead of 
often only $5 \mathrm{~mm}$. That concerns not only final positions of components but also temporary clearances during handling and maneuvering. The optimized design of W7-X, in particular its coils and vessels, required special threading equipment that allows a simultaneous coil maneuvering in all 6 degree of freedom. Fig. 1 shows a typical coil threading process. Moreover, planar and non-planar coils must be threaded into each other, what makes the tooling even more complex. The development and procurement of the threading equipment for the coils lasted three years in close cooperation with the industry. The tool-design reached its feasibility limit. A position accuracy of the coils of about $1.2 \mathrm{~mm}$ was achieved. Coils are bolted to the central support ring and they are supported against each other with additional support elements. What makes these attachments and bolts so tricky is that all these supports are tightened or welded after the coils have already been accurately aligned. Comprehensive 1:1 mockups were needed to develop processes to keep the displacements under control. Molding procedures were developed that made sure that massive keys and fitting wedges could be installed with an accuracy of up to some hundredths millimeters.

Another field of development were about 200 electrical joints used to connect the Cable in Conduit Conductor ( $\mathrm{CiCC}$ ) ends at coil-terminals and bus bars. Every electrical joint must be removable to enable the (theoretical) replacement of a coil. About two years time was needed to develop a robust design with sufficient margin in the transition resistance (achieved values $<1 \mathrm{nOhm}$ ) and in the pressure strength (achieved values up to $170 \mathrm{bar}$ ). As the conductor conduit is made of aluminum and the joint housing is made of steel, the welding of the individual joint-components required specialized procedures. Every weld was stressed with liquid $\mathrm{N}_{2}$ and leak tested. That applies also to the Helium pipe system, which consists of about two thousand single pipes. Altogether, the cryogenic components at W7-X contain some thousand welds, which were tested as described above. Nonetheless, one cold leak with about $5 \times 10^{-5} \mathrm{mbar} \cdot 1 / \mathrm{s}$ was identified in the commissioning, which, however, is by far small enough to have no influence on the operation of W7-X. An extraordinary challenge is the High Voltage (HV) insulation at the joints. Joints are situated in several layers in so-called connection domes on the cryostat vessel with limited access. The usual wet-wrapping procedure with Kapton ${ }^{\circledR}$ layers underneath proved to be the most practicable approach after some tests and under consideration of the available space and the available time. However, the position stabilization of the slippery epoxy resin/fabric layers required customized molds during the Room Temperature (RT) curing. In addition, several voltage taps per joint had to be integrated in the insulation, which required additional mechanical stabilizers. The robustness of the joint insulation process was limited. Therefore, each joint insulation was individually tested under Paschen conditions. Only a few insulations had to be re-worked. Fig. 2 shows the setup. However, in the commissioning of W7-X, two out of seven TFcoil circuits did not show the expected HV strength under Paschen conditions. With the use of additional sensors and a reduced peak voltage during fast discharges, safe operation of
W7-X has nonetheless been ensured. Up to now, none of the well-known insulation tests nor sophisticated irradiation methods were effective enough to localize these weak points in the insulation [6 and 7]. A more robust approach for similar insulation-challenges in the future could be to develop premanufactured insulation-parts for a simpler installation. An attempt in that direction, however, could not be finalized within the given timeframe. The design of bus bars, joints and the Hepipe system would have been much easier if the coils would have been manufactured in the flip-symmetric geometry of W7$\mathrm{X}$, i.e., all coil-terminals and all pipe-connections at the upper side of the machine and not 10 times alternating between top and bottom. In addition, the assembly process would have been much easier and less risky if joints would be arranged in straight upright position with sufficient gaps and not stacked in layers.

The installation of current leads (CL) for the coils was extraordinarily effortful. They are designed in pairs as pendulums with a mechanical pivot support at the warm bottom end. The top end is connected to the cold bus-bar system. The cryostat dome envelops the CL and forms the mechanical support. The electrical connection at the top end can only be made as long as there is no dome installed. On the other hand, the mass of deadweight of $2 \times 250 \mathrm{~kg}$ must be precisely kept in position during the electrical installation. Fig. 3 shows the arrangement of the CL. When the deadweight of the CLs is transferred to the support, any stresses in the electrical connection must be avoided. The development of this assembly procedure lasted for three years. Again, a special set of tooling was developed and tested in a 1:1 mockup. Complex welding procedures and soldering procedures also had to be developed. In future application it is worth the effort to reconsider this design solution. An arrangement of the CL on the upper side of the machine could lead to simpler assembly procedures. Of course, as a consequence, power supplies and RT bus bars must be positioned on the top of the machine.

The installation of about 250 ports at W7-X lasted about two and half a years. Ports have a mass between $50 \mathrm{~kg}$ and 1.5 tons with a wall thickness up to $15 \mathrm{~mm}$. The process qualification for the maneuvering of ports in all 6 degree of freedom, for the individual preparation of every port (cut to length and shape) and for the welding procedure lasted altogether about three years. Fig. 4 shows the needed alignment-equipment, the socalled port assembly bridge. Two towers contain elevators, which enable the vertical positioning of port assembly ramps. A steel bridge connects both towers with each other. One ramp can be moved on the bridge. The whole bridge can be moved on air cushions as a whole or each tower separately. First approaches for the cutting of ports to length and shape with simplified processes were too time-consuming or not successful (e.g. taking the as-built contour through trial installations or the control of the welding with simple laser pointers fastened at the ports). At the end, the best approaches were the intensive use of metrology for the as-built survey and the deduction of the needed geometry through reverse engineering. In addition, the welders used laser tracker surveys to control both the path sequences and the welding energy and they peened the weldseams to correct port positions. The typical assembly accuracy 
of ports lay between 2 and $4 \mathrm{~mm}$.

The basic machine consists of five pre-assembled modules, with 100 tons each. Fig. 5 shows one magnet module during transportation. The plasma vessel is suspended temporarily in the coil setup. Magnet modules were put in the bottom module shell of the cryostat and moved to their final position on the machine base in the experiment hall. Once the upper shell was added and welded, the ports were installed. Every module, when complete, was immediately connected with its neighboring module. This "in series" assembly saved up to one year on the critical path. Since individual modules were immediately end-positioned all completion work at every module could start at the same time (in-vessel, ex-vessel services, current leads, diagnostics), without waiting for the completion of all five modules in separate park positions. (The original assembly concept was to move all five modules simultaneously towards the machine center to ensure the best possible accuracy.) The single module positioning provides the advantage to minimize the impact of individual coil deviations onto the overall magnet field accuracy. Every module gets corrected alignment coordinates taking into account the as-built position of the already aligned modules (coils). At the end, the superposition of all five module/field proportions yields a minimum in the resulting total field deviation. That correction procedure was already part of the very early assembly ideas of W7-X. The topology of the magnetic field of W7-X was measured to an accuracy of better than $10^{-5}[1]$ and found to be accurate to better than $10^{-4}$ [2] compared with the pure geometrical alignment accuracy of individual coils of about $1.2 \mathrm{~mm}$ (coil diameter is about $3.5 \mathrm{~m}$ ).

From the schedule point of view, the in-vessel assembly was foreseen to start immediately after the first module positioning. On the other hand, the plasma vessel of $\mathrm{W} 7-\mathrm{X}$ gets its final stiffness only when all five modules are welded together (deviation in the vessel contour and position of less than $3 \mathrm{~mm}$ ). To make best use of the opportunity, the project started the installation of less accurate in-vessel components like bolts, brackets and some pipes. The assembly teams positioned and welded some thousands of these elements by means of two robots or through manual alignments, supported with measurement arms. Once the first two modules had been connected, the local area around this connection became mechanically stable, i.e., deviations were below $0.5 \mathrm{~mm}$. More accurate in-vessel components such as heat shields and panels were installed later. The components requiring the highest accuracy, in particular the test divertor unit and the baffles, were not installed. These elements were not needed for the first plasma. On the other hand, this approach shortened the critical path towards the first operation phase by a year. One major lesson learned from the first in-vessel installation concerns the stability and reliability of the used geometrical reference system. About 70 fiducials attached to the vessel wall represent the W7-X coordinate system in every module. The W7-X coordinate system, represented through about 150 fiducials in the walls of the experiment hall, forms the reference of the invessel system. Vessel modules deform when they are welded together. Hence, the reference system in every module changes as well. After the welding, the in-vessel system was referenced again and a best fit to the previous one was made. The installed components show a statistical deviation of up to $2 \mathrm{~mm}$ within this updated inner system. This system, in turn, deviates systematically from the outer W7-X coordinate system (locally up to $4 \mathrm{~mm}$ ). Deviations in this magnitude, however, do not noticeably influence the heat flux onto the divertor (at least with the present knowledge). For the appraisal of the achievable accuracy of a certain in-vessel component, one must add the individually achievable accuracy and the systematic deviation. Late clashes can occur if the clearances between successively installed in-vessel components lie in the range of only few $\mathrm{mm}$. This can noticeably increase the customization effort.

\section{COMPLETION OF IN-VESSEL COMPONENTS IN THREE STAGES}

The first Operation Phase of W7-X, OP1.1, lasted for about 12 weeks after the basic machine commissioning. The main purpose of OP1.1 was to demonstrate the functionality of all main machine systems (magnet system, vacuum systems, cryo system, first part of the cooling systems, ECRH heating, first set of diagnostics, as well as instrumentation, control systems and safety systems). Magnetic flux surface measurements confirmed the expected field accuracy of at least $10^{-4}$. The magnet system was energized up to a magnetic flux density of $2.5 \mathrm{~T}$ on the plasma axis. Several hundred sensors at the magnet system monitored the mechanical integrity of the complex support structure. In the plasma vessel, limiters instead of a divertor were used to enable the creation of first low-energy plasmas and to carry out first plasma experiments. The results exceeded the expectations. The operation team carried out about 1000 plasma shots with energies up to four MJ.

After OP1.1 a sequence of three completion phases (CP1.2a, $\mathrm{CP} 1.2 \mathrm{~b}$ and $\mathrm{CP} 2$ ) began with two specific embedded operation phases (OP1.2a, OP1.2b). The main aim of OP1.2a was to operate an inertial cooled Test Divertor Unit (TDU) and to determine hot spots caused by misalignments or asymmetries in the magnetic field. The data gained should be used later to optimize the position of the High Heat Flux Divertor (HHF). This approach minimizes the risk of thermal overloading of the very complex HHF. In OP1.2b the effect of so-called Scraper Elements (SE) was examined. These elements were installed in front of the TDU. It was confirmed in OP1.2b that these SE could be used to limit thermal peak loads on specific divertor areas in certain plasma configurations. CP2, eventually, finalizes the planned scope of construction of the W7-X project with the installation of the actively cooled HHF and the completion of the cooling of all in-vessel components. In every completion phase, the number and scope of diagnostics are further enlarged.

The first completion (CP1.2a) started in March 2016 with the installation of the TDU and the associated baffle modules. In addition, about 40 diagnostic systems were installed; $280 \mathrm{~km}$ of cables and a first expansion of the cooling circuits were laid. This assembly phase lasted 14 months and consumed about 100,000 direct person-hours. New recruitments were needed since all industry personnel involved in the first assembly phase 
were sent back to their firms after this mission. In addition, the construction-oriented institute organization was altered into an operation-oriented one. Comprehensively experienced and well skilled personnel of the Assembly Division filled gaps in the new organization. I.e. fifty percent of the personnel for CP1.2a had to be newly trained. That was a severe challenge for the ambitious CP1.2a schedule. In terms of the technical achievements, the TDU reached an accuracy of about $1 \mathrm{~mm}$ with respect to the in-vessel reference system. Steps between neighboring TDU-modules were limited to $<0.3 \mathrm{~mm}$ (to avoid leading edges). That yielded, together with the magnetic field accuracy, a "favorable power load distribution on the divertor with acceptable asymmetry" (citation of the project director). All CuCrZr cooling structures of the first wall are covered with 8000 customized carbon tiles as shown in fig. 6 . The complete scanning of the as-installed surfaces and the reverse engineering of the associated CAD data formed the basis for this customization. For the TDU installation, a complex invessel tool was developed and tested together with industry firms within one year. This forms the basis for all future divertor works. Work in the helically bent and narrow vessel is ergonomically stressful. Particularly, the safe handling of the target modules of the divertor weighing up to $70 \mathrm{~kg}$ is a continuous challenge.

After CP1.2a the operation of W7-X was successfully continued until December 2017. In a second short completion phase, called CP1.2b, scraper elements [3] were supplemented in front of two of the 10 TDUs. Well prepared and mocked up in advance, the installation proceeded as planned. The preparation of the access to the plasma vessel required almost the entire assembly time of about three months (warming up the magnet system, venting the cryostat and plasma vessel, installing the step protection onto divertor modules etc.). This applies also to all future accesses to the plasma vessel of W7-X and must be considered in the planning of maintenance phases or further upgrades. The operation with the TDU continued thereafter until November 2018. Again, the operation results exceeded the original expectations [4]. Well-controlled plasma pulses with $5 \mathrm{MW}$ ECRH over 30 seconds were achieved, corresponding to $150 \mathrm{MJ}$ heating energy. These results confirmed also the achievements in the installation of the TDU. There is no necessity to develop more accurate assembly procedures for the later HHF divertor, what would have been a significant technological risk.

In November 2018 the last completion phase of W7-X (CP2) started. This upgrade will enable a heating energy of $18 \mathrm{GJ}$, i.e., $10 \mathrm{MW}$ for 1800 seconds. For that, the TDU is replaced with the actively cooled HHF divertor and, in addition, 10 cryopumps are installed. Customized pipe sections connect the pipe-feedthroughs with the 12 target modules of each of the 10 HHF divertors. These pipe sections must be installed prior to the target modules. In addition, the divertor cooling needs a very comprehensive ex-vessel cooling pipe system. The very narrow conditions in the PV compel the mockup simulation of every single assembly step and require a 1:1 PV module mockup. Two more years were needed for the technical enhancement of the in-vessel tool for the safe and precise positioning of divertor modules. Fig. 7 shows the procedure: The positioning device holds the deadweight of the target module whereas the technician adjusts the device in all 6 degree of freedom. At the end of this process, self-centering fitting elements like bolts and latches ensure the accurate position of the target module.

Due to the increased heat load, additional heat protection measures are needed. That concerns actively cooled wall protections of ports (86 so-called port liners) as shown in fig. 8 , the closure of gaps between in-vessel components [5] and the creation of a new divertor closure. The step protection of target modules requires particular attention since assembly personnel must step onto target modules during the installation works. For the time being, the works go on as planned to fix all these challenging issues. About 200,000 direct person-hours are needed for the completion phase CP2.

\section{CONCLUSION}

$\mathrm{CP} 2$ progresses as planned. However, the originally estimated completion end (December 2020) had to be extended to July 2021. The main reasons for these delays were the immaturity of assembly processes and of component-designs at the time of the original estimate, and modified prerequisites for the hiring of industrial personnel.

Several general lessons were learned from the construction of W7-X: The assembly of W7-X would have been easier if more attention had been paid to the mount ability in the design of the components. Assembly-friendly arrangements of components and sufficient clearances between neighboring components could noticeably safe money and time. Doable assembly tolerances for every assembly step must already be considered in the design of the components. The gradual maturity of the design of the components requires parallel resources for the qualification and, at the same time, for the execution of assembly works. Own competencies in engineering (assembly technology) and planning (assembly scheduling and work preparation) in the Assembly Division are imperative for a fast and effective workflow. The standardized and streamlined preparation of work packages and associated assembly documents are indispensable prerequisites for a fluid execution and full traceability of the work, and a reliable quality assurance. A comprehensive Quality Management System and independent quality inspections during the course of construction are necessary. A weekly or bi-weekly updated resource-loaded planning enables early indications of bottlenecks and early and effective mitigation measures. A clear hierarchical organization together with a clear division of tasks and responsibilities, and key persons with high competence in management and engineering enable a forward-looking risk identification and risk mitigation, and a fast decision-making. The comprehensive involvement of industrial partners in all areas of the construction enable a flexible and efficient answer to frequently changing resource loads.

\section{ACKNOWLEDGMENT}

This article summarizes and supplements the content of a 
plenary presentation held on the 28th IEEE Symposium on Fusion Engineering, June 2-6, 2019, Ponte Vedra Beach, FL., USA.

\section{REFERENCES}

[1] T. Sunn Pedersen et al., "Confirmation of the topology of the Wendelstein 7-X magnetic field to better than 1:100,000", Nature Communications (2016), DOI: 10.1038/ncomms 13493

[2] S. Bozhenkov, M. Otte, C. Biedermann, M. Jakubowski, S. Lazerson, T. Sunn Pedersen, R. Wolf, and the W7-X Team, "Measurements and correction of the 1/1 error field in Wendelstein 7-X", Nuclear Fusion 59026004 (2019)

[3] Jeremy D. Lore (ORNL), "W7-X scraper element design and operation", presented at the $28^{\text {th }}$ IEEE Symposium on Fusion Engineering, Ponte Vedra Beach, Florida, USA, June 26, 2019

[4] Hans-Stephan Bosch (Max Planck Institut für Plasmaphysik), "Status of W7-X and the way to steady state operation", presented at the $28^{\text {th }}$ IEEE Symposium on Fusion Engineering, Ponte Vedra Beach, Florida, USA, June 2-6, 2019, ID280

[5] Jiawu Zhu (Max Planck Institut für Plasmaphysik), "Backside protection for Wendelstein 7-X In-Vessel Components", presented at the $28^{\text {th }}$ IEEE Symposium on Fusion Engineering, Ponte Vedra Beach, Florida, USA, June 26, 2019

[6] J. Baldzuhn, H. S. Bosch, K. Gallowski, H. Grote, T. Klinger, C. Kopplin, M. Nagel, K. Rummel, T. Rummel, K. Risse, F. Schauer, O. Volzke, L. Wegener, A. Werner (Max Planck Institut für Plasmaphysik), "Spark detection and search for high-voltage Paschen leaks in a large superconducting coil system", Proc. 28th International Symposium on Discharges and Electrical Insulation in Vacuum ISDEIV (2018), Greifswald, IEEE p. 107, IEEE Transactions on Plasma Science, doi: 10.1109/DEIV.2018.8537120

[7] L. Brooke Spreen, "Investigation of Discharge Inception on W7-X Superconductor Joint Insulation”, ETH Zürich, High Voltage Laboratory, Switzerland, Semester Thesis, spring 2017 


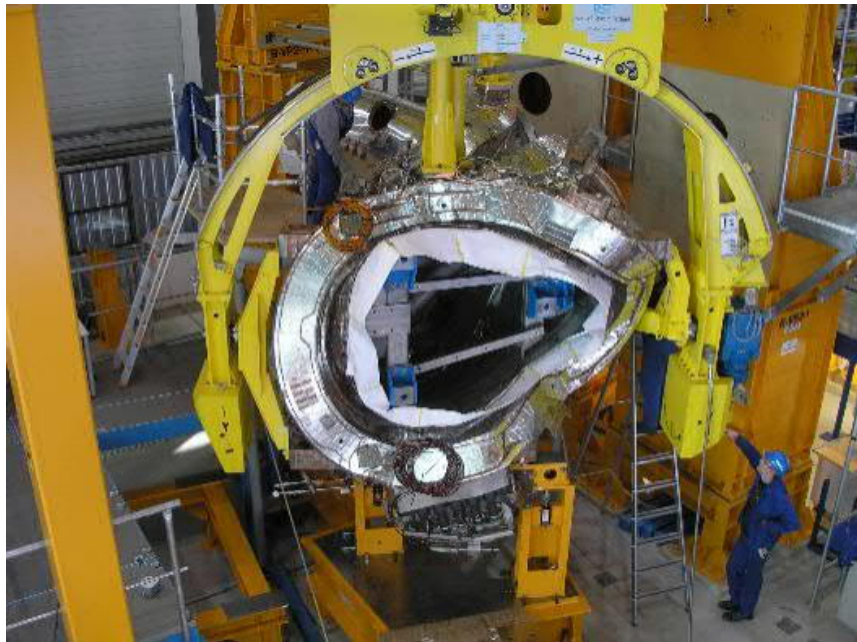

Fig. 1. Threading a non-planar coil across a segment of the plasma vessel

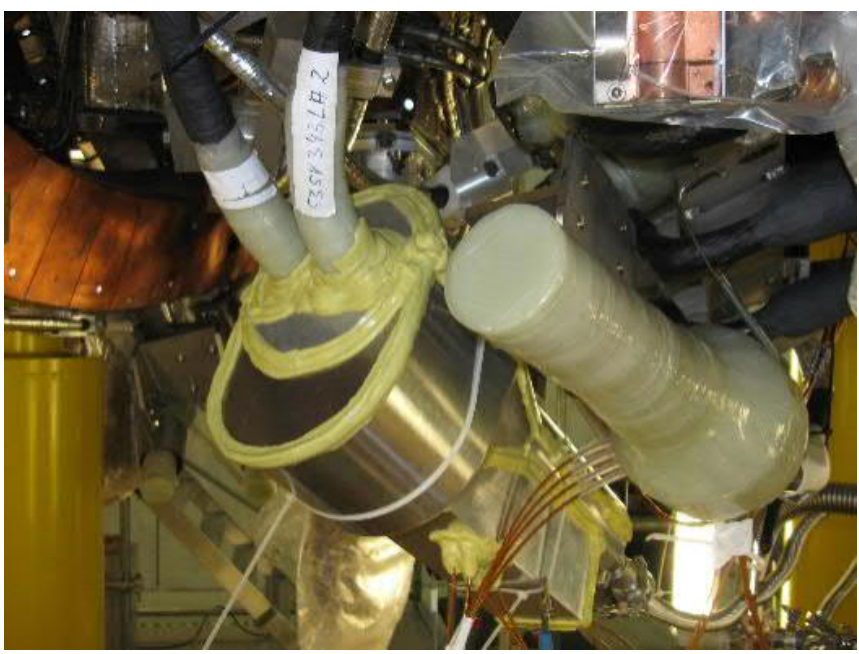

Fig. 2. Two insulated joints. At the left, a test chamber encloses the joint for local Paschen tests.

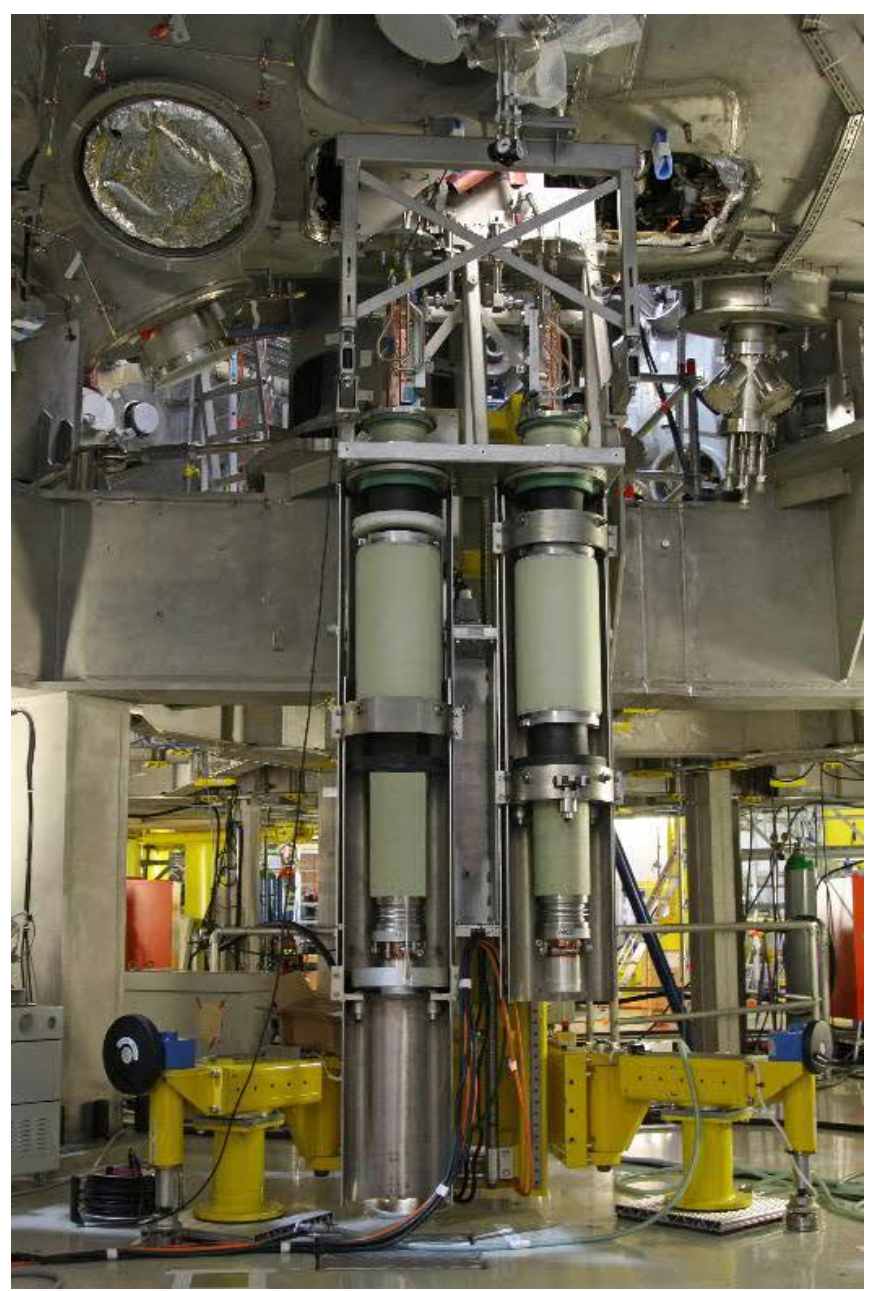

Fig. 3. The installation of current leads (feeders) at the bottom side of the machine. A modified port assembly ramp on air cushions holds both leads in position. The massive steel base carries W7-X with its 1000 tons.

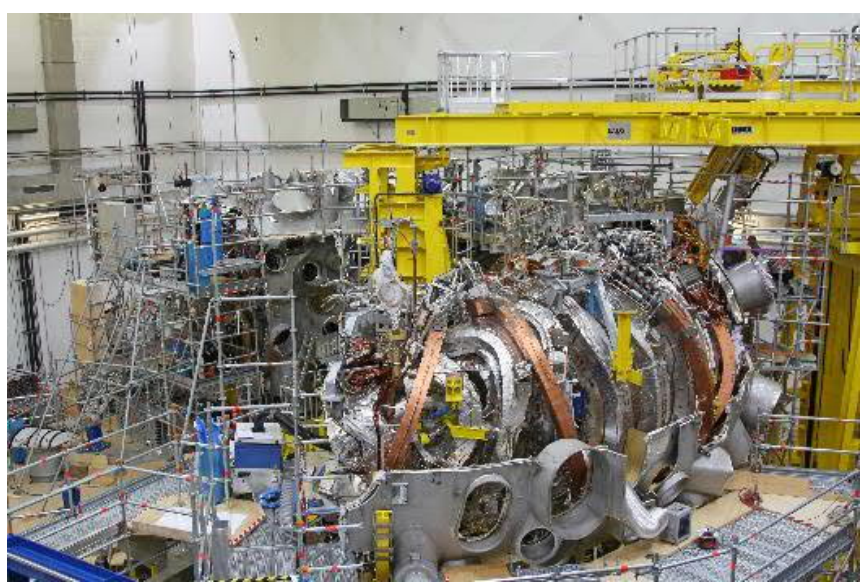

Fig. 4. Three modules in different completion stages together with the Port Assembly Bridge (yellow). One Port Assembly Ramp maneuvers a port into the machine from the bridge position. 


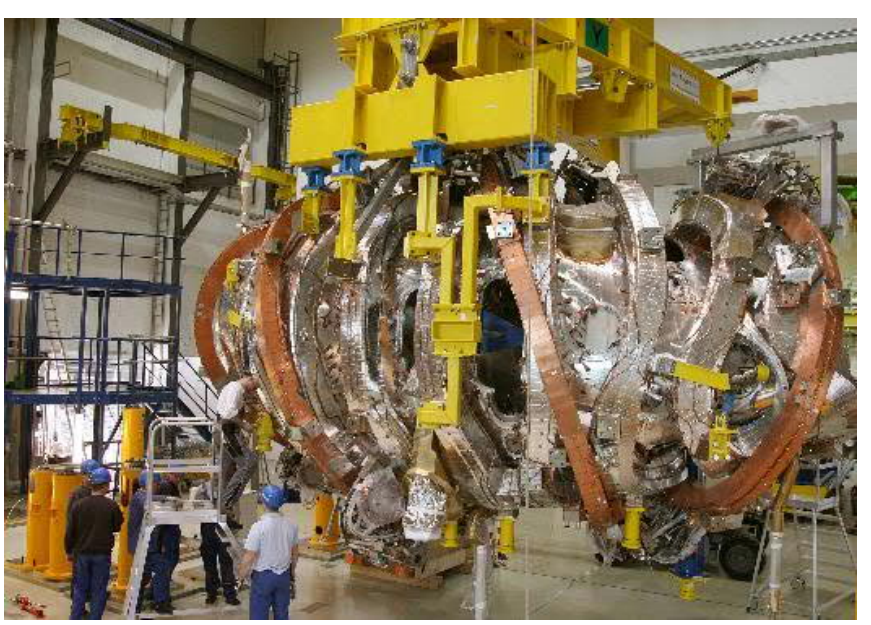

Fig. 5. A magnet module during transportation

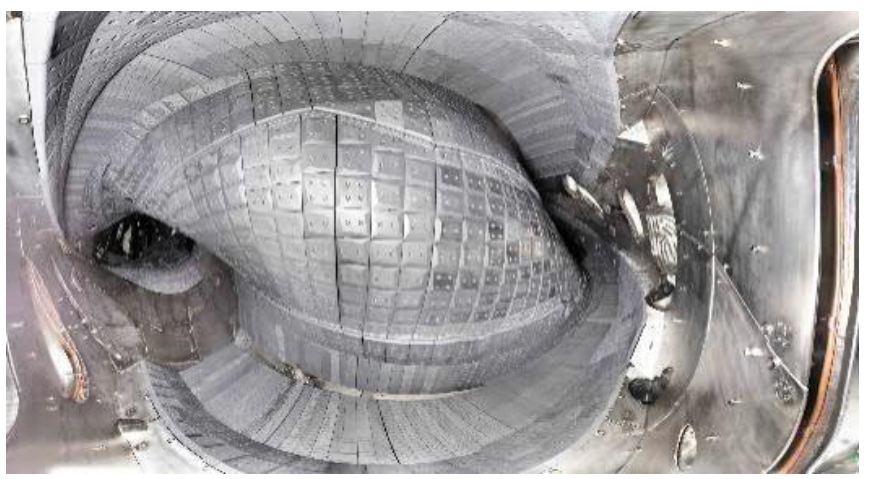

Fig. 6. Fish eye view in the plasma vessel with the completed TDU. Below in the middle of the photograph one sees horizontal and vertical target modules with the sickle-shaped pumping gap in between.

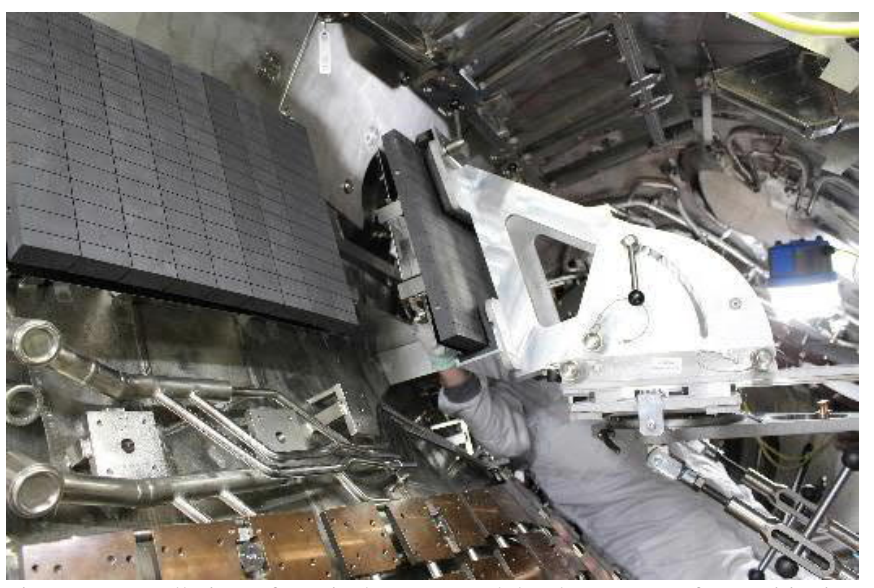

Figure 7: Installation of vertical target modules by means of a positioning device.

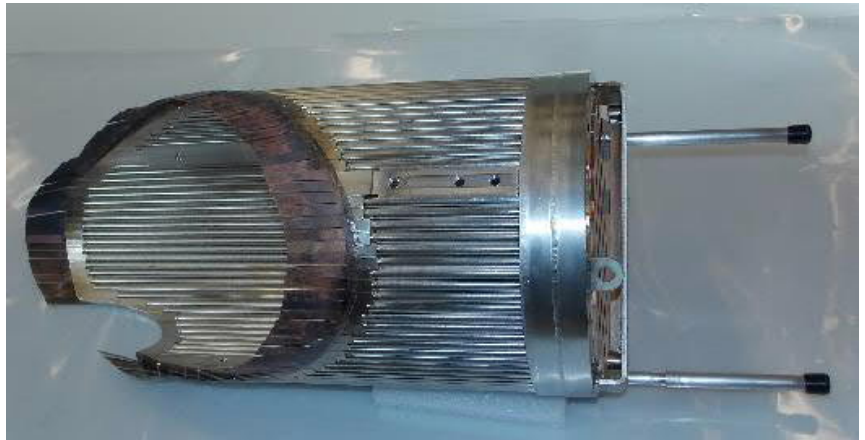

Fig. 8. Actively cooled port liners protect the port wall from heat radiation of the plasma (courtesy NTG Neue Technologien GmbH) 